\title{
Protée
}

\section{Vectoriser le désir. Réflexions sur une analyse impossible}

\section{Patrice Pavis}

Volume 27, numéro 1, 1999

La Mort de Molière et des autres

URI : https://id.erudit.org/iderudit/030533ar

DOI : https://doi.org/10.7202/030533ar

Aller au sommaire du numéro

Éditeur(s)

Département des arts et lettres - Université du Québec à Chicoutimi

ISSN

0300-3523 (imprimé)

1708-2307 (numérique)

Découvrir la revue

Citer cet article

Pavis, P. (1999). Vectoriser le désir. Réflexions sur une analyse impossible. Protée, 27(1), 9-16. https://doi.org/10.7202/030533ar

\section{Résumé de l'article}

Avec La Mort de Molière, on examine les limites d'une sémiologie classique fondée sur les signes et les structures observables et on propose en complément une analyse vectorielle attentive aux rythmes énergétiques et pulsionnels de l'oeuvre filmique. On plaide pour un dosage des deux méthodes : la vectorisation sémiologique, qui exige un repérage formel des signes, des vecteurs de sens, des continuités narratives ou formelles, des variations et des relances, et la vectorisation du désir, qui interroge le spectateur sur ses perceptions kinesthésiques, rythmiques, énergétiques, aussi sur ses désirs conscients et inconscients, ses puncta et ses vectores, autant de facteurs qu'il est difficile d'objectiver pour le regard scientifique. 


\section{- VECTORISER LE DÉSIR? RÉFLEXIONS SUR UNE ANALYSE IMPOSSIBLE}

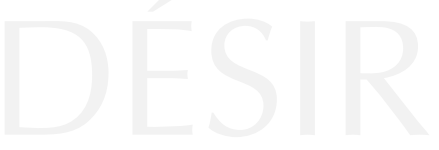

PATRICE PAVIS

En choisissant, avec Eli Rozik et Rodrigue Villeneuve, La Mort de Molière comme l'objet de notre projet de recherche, on pouvait être sûr au moins d'une chose: aucun individu, aucune équipe armée des plus puissants logiciels, ne parviendrait jamais à interpréter, expliquer, ni même à analyser cette vidéo de haute définition. N'était-ce pas là un sujet idéal pour un numéro de revue? Il nous permettrait peut-être de faire le point sur la méthodologie de l'analyse des spectacles (théâtraux, filmiques, vidéo). Cela nous conduirait-il nécessairement à récuser toute interprétation comme arbitraire et réductrice, à suggérer que toute théorie est désarmée devant une œuvre ouverte à toutes les pistes de lecture, et donc à renoncer à toute observation vérifiable?

"Ce n'est pas un poème sur Molière», annonce d'entrée la vidéo par la voix d'une petite fille disant un texte d'Heiner Müller. On est alors tenté de répliquer: "Ce n'est pasune étude herméneutique sur La Mort de Molière, une analyse explicative des thèmes, des motifs, des signifiés». C'est plutôt une opération destinée à utiliser Molière, cet "objet pour la médecine», pour poser la question méthodologique suivante: à l'analyse sémiologique classique (étude des signes et notamment des signifiés) doit-on substituer, en douceur, en "fondu enchaîné», un modèle énergétique; à défaut d'une énergétique ${ }^{1}$, du moins une «vectorisation du désir " 2 ? Une telle vectorisation du désir devrait alors tenir compte à la fois des vecteurs ou des formes objectivement observables dans le film et du désir de voir et de comprendre ce que l'observateur projette sur l'œuvre. Il s'agirait de remplacer une herméneutique de l'œuvre s'offrant à l'observateur sous forme d'un sens à décoder par une vectorisation de l'imaginaire et du corps de l'observateur, lequel serait, pour ainsi dire, mentalement et corporellement transporté par le défilement de la vidéo.

Transporté, le spectateur l'est au sens physique et kinesthésique du terme: il suit le mouvement, entraîné par un rythme narratif, musical, verbal, filmique (montage) et visuel (événements moteurs dans le plan). Un parcours le mène de surprise en surprise, de relance en relance, de choc en choc. Mais comment mesurer ces transports? Comment les sortir du langage métaphorique pour les prendre à la lettre? Y a-t-il un sismographe dans la salle? Ou du moins dans notre corps? Est-il fiable et que lui demander? 
Peut-être cette chose très simple: comment passe-t-on d'une situation initiale à la conclusion du film, depuis le tableau de Molière en César, transporté par une petite fille qui se cache derrière lui, jusqu'au tombeau qui se referme après que le mort soit parti en fumée? Il s'agit de mesurer la tension globale de cet arc qui constitue la fable. Cet arc est tendu aussi par notre volonté de retrouver une logique narrative à tout prix. Il offre un premier cadrage dramaturgique à

l'intérieur duquel on reconstitue une chaine d'actions et de séquences narratives. Dans tout objet esthétique, même un caillou devant nous baptisé sculpture, il y a toujours une volonté narrative et, pour l'observateur, un désir qu'on lui raconte des histoires.

Pour mesurer la tension de cet arc narratif, on effectue un repérage et un découpage des séquences, en partant non pas tant du scénario publié que de la réalité du montage. Il est facile de distinguer dix séquences thématiquement différentes: 1) exposition ("Molière se meurt»); 2) Colbert, Racine, le Roi; 3) les acteurs; 4) le docteur; 5) le père; 6) l'enfant et la claveciniste; 7) le renard; 8) Madeleine et Armande; 9) les manuscrits brûlés; 10) la mort.

Cet arc narratif révèle une continuité temporelle et une progression, même si l'ordre des séquences est arbitraire. Elles comprennent, en effet, autant de souvenirs évoqués par Molière que de scènes des personnes assistant à son agonie: l'image et le récit ne font pas la différence entre évocations fantasmatiques et scènes réelles, sauf peut-être dans la plus grande lenteur des premières ( $8 \mathrm{e}$ et $9^{\mathrm{e}}$ séquences par exemple). L'ordre des dix séquences n'est ni logique, ni chronologique: les scènes remémorées ou jouées sont empruntées aux lieux communs sur la vie et l'époque du dramaturge. Le spectateur y retrouve des souvenirs d'école, des représentations stéréotypées de la vie à la cour, sans qu'aucune révélation ne vienne surprendre ses attentes. D'où l'évaluation très contrastée que les récepteurs font de ces épisodes: certaines chutes de tension dramatique produisent des chutes d'attention chez le spectateur, et inversement. Mais quelles que soient ces variations, tantôt objectives, tantôt subjectives, de la tension (et de l'attention), l'arc narratif de la fable est organisé en fonction de la mort: tout y mène, par intensification ( $2 \mathrm{e}$ séquence) et par accélération (10e séquence), tout se termine sur une montée dramatique, un crescendo et une coda de la musique répétitive de Philip Glass et des principales représentations culturelles de la vie de Molière reprises une dernière fois.

En mesurant la tension de l'arc narratif, on s'efforce de ne pas disjoindre les matériaux (musique, cadrage et montage, voix, textes lus, etc.). On ne sera pas tenté de voir l'image comme mise en scène des textes, si l'on se souvient que les textes ont été choisis, écrits ou enregistrés par Müller et les collaborateurs de Wilson après le tournage et le montage de la vidéo. Le rapport entre l'image et le texte est toujours allusif et non explicatif; il génère une série d'ancrages possibles de l'un avec l'autre, sans qu'aucun des deux ne prenne jamais définitivement le dessus, mais toujours en résonance.

Pour évaluer la tension narrative globale, il faut aussi établir la tension et la tendance de chaque séquence en soi, comprendre la vectorisation de tout segment. La vectorisation ne s'effectue pas seulement par accumulation (confirmation d'un signifié ou d'un signifiant et condensation en de nouveaux signifiés ou signifiants), elle est le plus souvent faite par connexion et déplacement de l'élément significatif qu'il faut retrouver comme le motif refoulé du rêve. Ainsi au plan 23, on voit Molière faire des gestes convulsifs des mains, puis le plan 24 enchaîne sur les figurines de la commedia dell'arte, en insistant sur leurs jeux de mains, tandis qu'on entend un passage de Dom Juan où le personnage éponyme est accusé de tous les vices, notamment celui d'être "un épouseur à toutes mains». Enfin le plan 25 focalise à nouveau sur les mains de Molière. Ce déplacement du motif de la main, depuis les mains luttant contre la mort jusqu'aux mains légères de la vie théâtrale, alors qu'il est question de la main que l'on donne (pour épouser quelqu'un), travaille la métonymie de la main comme symbole du mariage. Molière se trouve rattaché à la vie, grâce à la main tenduevers Armande, mais il lutte contre la mort, 
lorsqu'il reçoit l'alliance mortelle (le scarabée) des mains de son ancienne amante.

À chaque séquence correspond une tension narrative, et une intention dramaturgique, que nous pouvons facilement reconstituer. Le survol en deux travellings successifs des quelques personnages expose, telle une liste des protagonistes au début de la pièce, les principales personnalités et étapes de la carrière de Molière (1 $1^{\text {re }}$ séquence). Le contexte historique ( $2 \mathrm{e}$ séquence) et théâtral (3e séquence) est évoqué dans le souvenir de Molière, tandis que la religion et la médecine se disputent le pouvoir d'aider Molière ( $4 \mathrm{e}$ séquence). Le médecin a remplacé le prêtre - nous dit le texte de Kafka, entendu en voix off au plan 31, mais, quand bien même il boit l'urine des patients dans un calice en forme de pot de chambre, le médecin ne peut plus rien pour le moribond. Même son père, tel Dieu interpellé par le Christ, est lui aussi contraint d'abandonner son fils. Il cire nerveusement le fauteuil et le bruit est celui d'un cercueil qu'on rabote ( $5^{\mathrm{e}}$ séquence). Tout le reste, la récitation des vers latins, la musique au clavecin ( $6{ }^{\mathrm{e}}$ séquence), les facéties du renard ou les conseils pour calmer latoux ne sont que d'aimables plaisanteries, des souvenirs plutôt agréables de Molière. Il y a là une véritable chute de tension, un remplissage textuel et filmique très décevant qui n'apporte rien à la fable dans son ensemble.

Par contre, l'arrivée très lente d'Armande, puis de Madeleine, constitue le sommet dramatique et émotionnel de la vidéo (8e séquence), ce qui relance la fin tragique: Molière brûle ses manuscrits (9e séquence), avant de disparaître lui-même en fumée, au terme d'une longue séquence de 23 plans, au cours de laquelle tous les thèmes sont repris dans un ballet funèbre orchestré par les religieuses (10e séquence).

Au plan de l'arc tout entier, comme à celui de chaque séquence, le spectateur joue un rôle capital pour vectoriser des éléments distincts, faire l'hypothèse de leur sérialité et de leur tension. Pour une première orientation, à l'intérieur de cet arc, on pourrait reprendre le fonctionnement général en quatre types principaux de vecteurs que nous proposions dans l'analyse des spectacles, en précisant comment ils s'organisent dans le cadre particulier de cette vidéo.

\begin{tabular}{|l|l|}
\hline $\begin{array}{l}\text { Métonymie } \\
\text { (Déplacement) }\end{array}$ & $\begin{array}{l}\text { Métaphore } \\
\text { (Condensation) }\end{array}$ \\
\hline Connecteurs (2) & Accumulateurs (1) \\
\hline Sécateurs (3) & Embrayeurs (4) \\
\hline
\end{tabular}

(1) Les accumulateurs rassemblent un nombre important de biographèmes - ces unités minimales obligées de la biographie de Molière -, d'actions légendaires et de représentations sociales: il s'agit plus d'un "comprimage", d'une synthèse nouvelle, que d'un collage ou d'un montage. L'accumulation d'objets en pleine décadence ou destruction fournit la matière condensée de ce comprimage.

(2) Les connecteurs mettant en séquence ces matériaux comprimés sont plus formels que thématiques: notamment rythmiques et musicaux. La musique répétitive de Philip Glass se prête à cette connexion, homogénéisation et intégration des matériaux les plus hétéroclites: le déplacement métonymique du motif confère à la répétition une plus grande dramatisation, laquelle est renforcée par les signes visuels de la mort imminente. Sans cet élément connecteur de la musique, les connexions visuelles, les relances, les leitmotive renvoient parfois à des plans précédents sans vraie nécessité (aussi les plans $49 \rightarrow 46$; $50 \rightarrow 46$; $51 \rightarrow 16 ; 54 \rightarrow 15$ ).

(3) L'effet de récit hagiographique ou tragique est ainsi sans cesse interrompu par des sécateurs qui entravent le flux du récit ou dérangent la perception attendue: bruit de verre cassé, crissement désagréable, «hum» ponctuant la souffrance de Molière, bruits auxquels s'ajoutent les coupures souvent imprévisibles qui terminent un plan ou une séquence ou encore retour régulier et régulateur du motif visuel du fauteuil, sur lequel commence à chaque fois un nouveau texte.

(4) Ces ruptures systématiques, qui morcellent le récit autant qu'elles le structurent, obligent le spectateur à embrayer sur un tout autre plan, à suivre la principale hypothèse herméneutique concernant l'énonciation globale de l'œuvre ou, si l'on préfère, le métatexte de la mise en scène: ceci est-il ou non un poème, à savoir autre chose qu'un «objet pour la 
médecine», et donc une idée, une œuvre vidéographique, un «tombeau littéraire» d'un auteur immortel? A-t-on le droit - telle est la question implicitement posée - d'embrayer depuis la matérialité des représentations filmiques sur l'idéalité d'une œuvre filmique en train de se faire?

La vectorisation s'observe également au niveau microscopique d'un ou de deux plans. Soit la scène où Molière, sur son lit de mort, agite convulsivement les mains, à la recherche d'on ne sait quoi (plan 24).

- (1) L'accumulation-condensation du sens pourrait être le signifié ambigu suivant: être à l'agonie, se raccrocher à la vie. Les mains «aboient» (mouvement et bruit vocal de Wilson), tandis qu'on entend un texte où Dom Juan est comparé à un monstre, à un «épouseur à toutes mains» («eine Heiraterbestie», dans la version allemande: une bête à épouser).

- (2) La connexion s'effectue ainsi entre des séries qui se rencontrent comme sous l'effet du hasard, avant d'immédiatement s'entrechoquer et diverger. - (3) Mais cette rencontre forcée, qui déplace, refoule et révèle bien des motifs, menace de rompre l'alliance du texte et de l'image, et du même coup l'unité de la personne humaine, et c'est ce qui est marqué par les aboiements de Molière qui coupent court à toute conversation, et a fortiori à toute idéalisation poétique.

- (4) Lorsque, plus tard, cette même main tentera de saisir celle d'Armande ou recevra le scarabée que Madeleine, comme cadavre vivant, dépose sur son majeur, le spectateur devra embrayer sur d'autres associations d'idées: celles qu'il trouve dans son expérience personnelle, car là encore nul ne saurait prétendre posséder la «bonne» interprétation.

À tout moment de la réception du film, que ce soit au cours de sa projection ou après en repensant à ses procédés, il est loisible au spectateur, à défaut de trouver le sens, de s'essayer à des vectorisations et de faire ainsi l'hypothèse de la construction possible d'une séquence plus ou moins large de l'œuvre. S'agitil du sens de l'œuvre? Souvent oui, mais pas toujours, ou pas uniquement, car il peut s'agir aussi d'une circulation de rythmes, d'énergies, de flux, de courants peu faciles à saisir et à conceptualiser, mais qui paraissent pourtant nous transporter à travers l'œuvre, sans que nous puissions nous opposer à la force qui les meut et nous avec eux.

Il convient donc de distinguer ces deux types, différents quoique complémentaires, de vectorisation: sémiologique et énergétique.

1) La vectorisation sémiologique travaille à partir des signes tangibles, signifiants comme signifiés; elle identifie les formes, les séries, elle navigue d'un plan à l'autre, elle connecte, associe, rompt, embraye sur des unités de sens, définit les vecteurs en fonction de leur origine, leur destination, leur trajectoire et leur vitesse. On a vu que la fable est assez étroitement vectorisée. On pourrait en dire autant du système de l'espace ou de la gestuelle: leur séquence de signes paraît clairement et dynamiquement orientée, chaque élément étant lié à une propriété formelle descriptible. Mais la réception d'une œuvre aussi dense et émotionnellement chargée ne se borne pas à cette vectorisation inscrite dans les formes et tracée d'avance. Elle doit aussi rendre compte de tout un courant d'émotions qui semble nous subjuguer, mais que nous ne parvenons tout d'abord pas à identifier et surtout pas à canaliser.

2) La vectorisation énergétique - puisqu'il faut l'appeler par son nom - n'est pas tant un parcours strictement défini qu'une suite d'intensités différentes. Elle n'organise pas les signes, elle fournit un convecteur d'énergie pour tout ce qui, dans le spectacle, est susceptible de toucher la sensibilité de l'observateur, notamment par les variations d'intensité, de tempo et de flux, tel un fleuve si chargé qu'il a oublié la trace de son lit et submerge toute la vallée. Elle ne se limite pas aux systèmes immatériels (comme la musique et le rythme), mais tend à absorber l'ensemble des systèmes de la représentation.

La première séquence, après l'introduction du portrait de Molière par la petite fille et le générique, à partir du plan 4 (travelling latéral), procède par exemple en trois lancements successifs dont la série fait l'effet de la suite d'un motif identique, selon une amplification-intensification. On entend d'abord la 
musique répétitive de Philip Glass, qui devient la basse continue de la scène, la vectorisation dominante (par son ampleur et son battement rythmique extrêmement précis) sur laquelle tout le reste pourra se greffer. Quelques secondes plus tard commence un travelling en gros plan qui défile à la hauteur du visage, voire des yeux seulement, de quelques-uns des personnages. Quarante secondes après, la voix de Heiner Müller récite, de manière volontairement objective et détachée, le texte sur Galilée qui «observe les étoiles", tandis que le fauteuil de Molière est installé sur le carré lumineux, sorte de Wunderblock, de bloc magique, image dont Freud se servait pour figurer les strates des fantasmes qui s'effacent les unes les autres dans la conscience du sujet.

La vectorisation énergétique est la résultante de ces vagues successives se déplaçant à des rythmes différents, avec des déphasages variables, selon une plus ou moins grande quantité d' «ondes» superposées. Elle emprunte sans la moindre gêne les réseaux existants, ceux que la vectorisation sémiologique est capable de détecter ou de tracer. Elle gère à sa manière l'énergie du mouvement, du rythme, de

l'intensification, du crescendo de la tension émotionnelle. Tout le film, en dépit de son apparence fragmentée et composite, est bâti sur un crescendo, plus émotionnel et musical que narratif ou thématique, lequel crescendo est maintenu en mouvement par l'armature très solide et précise des relances et des intensifications, jusqu'au moment final de la mort et de la mise au tombeau. Plus encore que la vectorisation sémiologique, c'est la vectorisation des intensités qui structure et organise l'ensemble de l'œuvre vidéo. Il n'est du reste pas aisé de distinguer les éléments objectifs, sémiologiques, et les flux plus labiles de l'intensification des énergies. C'est pourquoi on ne fera pas, dans cette énumération des propriétés de la vectorisation, de distinction entre le sémiologique et l'énergétique, on signalera seulement quelques-unes des fonctions observables dans cette vidéo.

1) La vectorisation est responsable autant de la structure narrative (les motifs de la fable) que de la structure rythmique et de l'impression d'accélération et d'intensification. Les deux structures toutefois ne coïncident pas: il semble que le rythme se serve de la fable comme d'un marchepied ou d'un tremplin pour trouver sa propre allure et déborder les formes prévues, de manière à tout emporter sur son passage, comme le fleuve sorti de son cours traînant avec lui une masse d'alluvions (de signes étrangers arrachés aux autres systèmes signifiants). C'est l'impression que l'on retire du crescendo qui conduit à la mort, après que, par des mouvements de caméra inhabituels, inattendus et peu soignés (plans 80 à 92), Wilson a réussi à donner une impression d'inéluctabilité, d'un moment où toutes les images d'une vie, à l'instant de la mort, se débattent en nous avant de sombrer.

2) La vectorisation est l'art de la relance, narrative et rythmique, notamment par la répétition du planleitmotiv sur le fauteuil ou de l'incipit «Molière se meurt». La relance est ponctuée par les ruptures visuelles (changement de plans) et les incidents sonores (bruits étranges non motivés par la situation de l'image).

3) La vectorisation pose des jalons, notamment sur des «questions d'école» comme: «Molière et son temps", "Molière et le théâtre", "Molière et les femmes». Chaque lieu commun part d'une évidence culturelle, connue de tous, et la développe jusqu'à ce qu'elle forme une unité narrative, contre laquelle d'autres représentations, à la suite d'une rupture formelle ou thématique, peuvent se développer.

4) La vectorisation se manifeste autant dans la macrostructure (narrative et rythmique) que dans la microstructure: à l'intérieur d'un plan ou d'un plan au suivant. Soit par exemple le plan 9: un travelling avant découvre la bouche de Molière crachant le sang; un fondu enchaîné, procédé filmique idéal pour la condensation de deux signifiants en un, nous fait passer lentement du sang à la robe rouge, "passionnée», d'Armande. Quel est le sens (c'est-à-dire la direction) de ce transfert, de cet embrayage d'un signifiant à l'autre (sang, robe)? Peut-être l'alliance de la mort et de l'amour: à l'heure de sa mort, son amour pour elle lui revient; il ne peut vivre sans elle, à la rigueur survivre encore un court instant. 
5) La vectorisation organise, dynamise et donc hiérarchise les signifiants, en décidant de leur connexion, de l'origine et de l'impact du vecteur. Chaque nouvelle séquence repart d'une autre origine, laquelle est connue dès la deuxième ou troisième occurrence; elle doit tout rebâtir de nouveau, même si la direction générale du vecteur narratif est déjà tracée d'avance: on sait où l'on va, ne serait-ce que parce que le titre nous a annoncé «la mort de Molière». Dans le détail, la connaissance toujours incertaine de l'origine et surtout de l'impact du vecteur oblige à respecter et à préserver la matérialité et la littérarité du signifiant, de ne se fier, pour l'analyse, qu'à la logique du signifiant. Tous ces signifiants sont réduits à l'état de matière inerte, détruite, en décomposition, à l'image du corps de Molière, du verre cassé, de la fumée s'échappant de la tombe. Comme s'il fallait réduire tout texte à de la matière, avant de pouvoir reconstruire la statue du commandeur, préserver l'œuvre écrite où la voix s'est inscrite, trace du corps disparu dans le texte immortel.

6) La vectorisation oblige ainsi le spectateur à tracer des voies dans la matérialité du film; à connecter l'inconnectable pour produire une fable plus ou moins orthodoxe et paradoxale, à découper à sa manière la matière signifiante, à traiter certaines parties comme de la matière pure, sans réduction possible à un signifié. Certains plans visuels très formels (les dalles grises, par exemple), ou «baroques» (l'alignement des protagonistes), ne peuvent et ne doivent pas être compris, mais appréciés comme du bruit, du rythme ou de la musique; d'autres plans, au contraire, fournissent, par leur répétition et leur ancrage immédiat à des signifiés des textes ou à la logique narrative, des clés pour une herméneutique qui nous rassure et nous encourage sur notre faculté et notre plaisir de comprendre. Mais quels plans et à qui d'en décider?

7) La vectorisation mène ainsi à une «écoute sensible» ${ }^{3}$, à une pratique du signifiant, de sa logique, ce qui encourage l'utilisateur à proposer diverses manières de vectoriser la matière signifiante, sans partir, du moins systématiquement, des signifiés suggérés par les textes ou la fable, ou d'un quelconque sous-texte, d'une symbolisation, d'une clé du sens, dont on s'imagine, qu'une fois trouvée, elle ouvrirait toutes les serrures.

8) La vectorisation énergétique est finalement comparable à une des quatre dimensions de tout mouvement selon Laban ${ }^{4}$, le flux. Dans le mouvement humain, le flux est moins objectivement observable et analysable que les trois autres dimensions, celles de l'espace, du temps et du poids. (1) Le poids correspond ici à la corporalité massive de Wilson/Molière, lourdement jeté sur son lit, dont aucun mouvement n'émane sinon parfois les mains devenues "folles", la bouche saignant, toussant ou criant. (2) L'espace est celui du champ de la caméra, il est donné morceau par morceau, sans souci de totalité (à part le plan récapitulatif 75 qui fixe théâtralement tout ce qui a été saisi partialement depuis tous les angles possibles). (3) Le temps est celui d'une dernière journée, dont on nous présente quelques moments forts correspondant à une interaction du monde extérieur avec Molière ou, sans que cela soit marqué dans l'image, à ses souvenirs. À part les moments de crise ou d'aboiement, le rythme corporel de Wilson est très lent, ce qui contraste avec celui «normal» des personnes évoquées ou accéléré des religieuses et de la musique de Glass qui accompagne les funérailles. (4) Le flux, comme le mouvement, est en somme la résultante des dimensions objectives de l'espace et du poids, et elle seule est en mesure de leur conférer une qualité individuelle et unique.

Ce flux global, plus ou moins canalisé par les formes objectives descriptibles, donne une image de ce que pourrait être le dispositif de l'énonciation de l'œuvre dans sa globalité. En effet, ces libres promenades dans la matière de la vidéo, auxquelles les vectorisations partielles et parfois fortuites nous invitent, se déroulent malgré tout dans un cadre strictement délimité, qui est celui du dispositif de l'énonciation. Ce dispositif qui englobe tout le film et ses énoncés (ses plans, ses séquences) nous paraît indispensable, si nous persistons à vouloir analyser le film dans son ensemble, comme un objet qu'il faut 
construire pas à pas, et qui finit par former un tout, à savoir la représentation vidéofilmique de la mort de Molière. Certes, la vidéo n'a plus d'instance auctoriale biographique bien définie, puisque chacun y a contribué, à des moments divers, sans tenir compte d'un plan d'ensemble: le choix des textes, des musiques, le montage définitif échappent au contrôle central d'une seule personne. Pourtant, étant donné que nous ne nous intéressons pas à Wilson metteur en scène ou auteur, mais à l'œuvre achevée comme objet esthétique de notre analyse, il nous paraît légitime de constituer cet objet en une totalité ayant son propre mode de fonctionnement et d'énonciation et de rendre compte de la manière «retotalisée» dont nous avons à un moment donné - et sans préjuger de l'avenir - pu «recevoir» cette œuvre.

Nous partons donc, dans cette analyse, d'une vision synthétique de la vidéo et de son énonciation globale. Ce qui nous autorise à poser les questions de l'énonciation et des énonciateurs: qui parle, qui émet ces images, ces sons, ces textes? Il faut là encore repartir des unités dynamiques, des vecteurs qui se mettent en place. Avant le générique, on entend les pas d'une petite fille portant devant elle un portrait de Molière qui la dépasse de beaucoup, par sa dimension, et par le texte affirmatif (de Müller), lequel commence par une dénégation (semblable à celle, célèbre, de Magritte: "Ceci n'est pas une pipe»): "Ce n'est pas un poème sur Molière». Ce prologue se termine du reste par cette formule inverse, à laquelle le spectateur débutant ne peut que souscrire: "C'est un poème sur Molière». La petite fille parle à la forme neutre: "On ne peut pas écrire un poème sur Molière. Molière n'est pas un sujet de poésie, Molière c'est un objet pour la médecine». Ce faisant, elle pose d'entrée la structure objectale et affirmative de la vidéo: C'est + objet, cet objet pouvant être verbal ou visuel, consister en un poème ou une image. S'ensuit en effet une énumération hétéroclite de propriétés générales sur Molière («c'est...»), puis une série, à la fois métaphorique (accumulative) et métonymique (connectrice) d'images qui représentent des moments connus de sa vie et de son époque: autant de personnes qui défilent devant lui (et devant nous) comme en son for et son théâtre intérieurs. Lorsque Molière finit par s'envoler en fumée (plan 77), comme ses manuscrits, le MOI a disparu, comme l'annonce le dernier texte de Heiner Müller: "Voulez-vous que je parle de MOI qui de qui est-il question quand il est question de moi QUI est ce MOI»(plan 79). Ce MOI parti en fumée, de nombreux éléments visuels, qui sûrement le composaient, sont repris en accéléré, selon une technique de "caméra déchaînée», qui contraste avec les plans très posés et statiques du reste de la vidéo. Ce sont là les dernières visions avant la mort, lambeaux de moi qui tournoient, après la disparition «magique» du Molière-objet-pour-la-médecine et sa transformation en une flamme du Saint-Esprit, comme s'il s'agissait du début du Molière «sujet de poésie», notamment de ce poème vidéographique de Wilson et de Müller. Car derrière ce portrait de Molière se cachent aussi son presque homonyme, Müller, et l'auteur de la vidéo, Wilson, lui aussi «acteur au travail qui représente un homme mourant appelé Molière». La mise en tombe de Molière, sa quasi-béatification dans les flammes, sur une musique qui s'apaise, scelle, avec la pierre de la tombe qui se referme, à la fois la fin de Molière, «objet pour la médecine» et la fin (et donc la présentation) du poème filmique. Ce que confirme l'apparition du générique, désagréable intrusion des remerciements dans le fictionnel et l'esthétique. D'autant plus qu'in extremis, alors que l'on croit tout fini, un dernier plan (95) de 14 secondes montre un ultime énonciateur, le médecin en costume contemporain, qui vient donner une dernière touche ironique en interpellant le spectateur, comme s'il s'agissait de savoir si la médecine a le dernier mot, même funèbre, ou si Molière est à présent sujet de poésie. La problématique objet/sujet, matérialisme/idéalisme, qui est un des thèmes transversaux de cette méditation vidéographique sur la mort, est ainsi mise en abyme dans le dispositif global de l'énonciation, dans la vectorisation qui nous conduit du corps-objet mourant à l'esprit-sujet immortel.

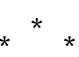

Y a-t-il encore une méthode universelle pour analyser des ouvres aussi complexes que La Mort de Molière, un 
sismographe, sensible et résistant, subjectif et objectif, capable d'analyser, enregistrer, interpréter, dessiner, caresser cet objet du désir et son sujet désirant, et ce à partir des vectorisations sémiologiques et énergétiques, dont nous sommes, en partie, mais en partie seulement, responsables? Nous avons ici plaidé pour un dosage des deux méthodes, la vectorisation sémiologique et la vectorisation du désir: la première exige un repérage formel des signes, des vecteurs de sens, des continuités narratives ou formelles, des variations et des relances; la seconde interroge le spectateur sur ses perceptions kinesthésiques, rythmiques, énergétiques, mais aussi ses désirs conscients et inconscients, ses puncta et ses vectores, autant de facteurs qu'il est difficile d'objectiver pour le regard scientifique. Mais une double difficulté méthodologique demeure: 1 ) repérer comment les changements de forme, la perception des signes, bref la vectorisation sémiologique vont produire chez le spectateur des réactions mentales et physiques descriptibles et mesurables; 2) trouver, pour toute expérience kinesthésique, rythmique, pulsionnelle, tensionnelle du spectateur, un fondement formel et vectoriel au sens d'une sémiologie descriptive de l'œuvre.

Pour accomplir ces tâches, il faut faire un usage beaucoup plus technique de notre sismographe, repérer par exemple les variations d'intensité et de tension dans différentes parties du corps de Wilson/Molière: son visage, ses mains, son buste sont soumis à des tensions musculaires différentes, ce qui affecte le spectateur de manière très variable: l'impassibilité du visage nous inquiète; les convulsions des mains nous étonnent et nous renvoient à l'objet invisible vers lequel elles tendent; le buste nous donne l'impression d'une masse immobile, d'un gisant qui ne peut plus toucher le monde extérieur qui défile devant lui. Il faut aussi établir un lien entre les tensions de ces gestes et la tension dramatique, cet arc narratif de la fable: ces jeux de mains de Molière se prolongent ainsi dans d'autres plans où il tend la main vers Armande, ou vers Madeleine; la tension musculaire et l'angoisse qui s'en dégage pour l'observateur sont prises en charge, relayées et modifiées par le récit. Mais à vouloir ramener ici l'intuition ou l'expérience kinesthésique du spectateur à une forme objective de l'œuvre filmique, et réciproquement, on risque de revenir à l'antique débat du sujet et de l'objet, voire de la forme et du fond, que précisément la vectorisation s'efforçait de dépasser. Nous sommes aussi ramenés, une fois de plus, à la question herméneutique du sens, une question que nous avions peut-être d'entrée un peu trop vite voulu enterrer. Car, ne nous faisons aucune illusion sur la faculté humaine de produire et de découvrir du sens en toutes circonstances: le signum et le studium sont toujours prêts à s'établir sur les traces vives du vector et du punctum. Et inversement, une vectorisation sémiologique (une analyse structurale donc) trop vite et trop lourdement tracée menaceà tout moment d'éclater, et ensuite de se perdre dans toute l'œuvre comme un ruisseau dans la plaine désertique.

En revenons-nous ainsi, selon la lourde fatalité de notre fin de siècle, au relativisme postmoderne, selon lequel nous pourrions tout dire, tout entendre dans notre «écoute sensible», et tout faire dans notre pratique du signifiant? Pas nécessairement, car même si nous sommes bien à l'origine des vectorisations de notre désir, nous n'en restons pas moins soumis à la loi impérieuse des formes, des signes, des vecteurs. Aussi la question n'est-elle plus tant, avec cette œuvre vidéographique comme avec le reste, de voir que d'oser voir, car, ici comme ailleurs, on ne sait pas trop ce qu'on ose voir et on n'ose pas trop ce qu'on sait voir.

\section{NOtes}

1. Au sens que J.-F. Lyotard donne à ce terme dans: «La dent, la paume", Des dispositifs pulsionnels, Paris, U.G.E., 1973.

2. Cf. P. Pavis, "Vectorisation du désir et travail du geste», L'Analyse des spectacles, Paris, Nathan, 1996, p. 64-90.

3. "L'écoute sensible commence par ne pas interpréter, par suspendre tout engagement. Elle cherche à comprendre par "empathie", au sens rogérien, le surplus de sens qui existe dans la pratique ou la situation éducatives"; R. Barbier, L’Approche transversale. L'Écoute sensible en sciences humaines, Paris, Anthropos, 1997, p. 294.

4. R. Laban, La Maîtrise du mouvement, Arles, Actes Sud, 1994. 